\title{
Analysis of Relay Deployment Based on Handover Outage Probability in High Speed Scenarios
}

\author{
Liang Jing, Xiaojie Xu, Yafeng Wang \\ Beijing University of Posts and Telecommunications, Beijing, China \\ Email: liangjing@bupt.edu.cn
}

Received July, 2013

\begin{abstract}
The LTE and LTE-Advanced systems are supposed to be the most popular cellular network in 4G networks. Relay technology is one of the most preferred technologies proposed in LTE-Advanced standardization to improve the capacity and coverage. This paper analyses the handover outage probability through relay deployment, and proposes some proofs and suggestions for relay deployment to minimize the handover outage probability in high speed scenarios.
\end{abstract}

Keywords: Outage Probability; Relay Deployment; Handover; High Speed Scenarios; LTE-advanced

\section{Introduction}

LTE-Advanced network, with one or more relays in one cellular, may be used to cover some high speed scenarios such as railway, highway and so on. As people are moving faster and faster via various vehicles, which brings up some challenges to security and reliability of the network, handover is becoming an important issue in those high speed scenarios.

Handover is usually based on signals transmitted from base stations (BS) or relay stations (RS) to mobile stations (MS). If MS finds out that the quality of signals from neighbor BS or RS is better than that from home BS or RS while other conditions are met, then MS releases channels in home cell and begins to use channels in the neighboring cell after a handover process. In high speed scenarios, signals transmitted in LTE-Advanced network not only experience shadowing and multi-path, but also suffer Doppler shift due to high speed. As a result, some solutions may be adopted to solve the problem. In previous work, handover algorithms are proposed to set different handover threshold [1-3] depending on different speeds. As we know, handover often occurs in overlapped areas, which is far away from BS, an accurate threshold can hardly be set to meet different speeds.

Relay technology can improve the coverage, enlarge the network capacity and reduce the energy cost. Of course, relay technology can also be used to assist handover in LTE-Advanced network. In LTE-Advanced network cells with relays, when MS decides to make handover decisions, it not only measures signals from neighboring BS and home BS, but also from RS. As a result, the deployment of RS is very important for handover. The position of RS may affect the outage probabil- ity and the stability after handover. In this paper, a novel analysis of handover outage probability based on signal strength in different relay deployments is made. Based on the analysis results, conclusions about relay deployment in high speed scenarios are made.

\section{System Model}

In cellular networks, there are two main deployments, which are regional coverage and linear coverage respectively. Regional coverage is usually applied in urban area, where people in wireless communication needs are distributed in a large area. For those with wireless communication service demands in high speed such as railway, linear coverage is preferred and should be applied. As showed in Figure 1, the center of round cells is in the same line with MS traveling along. The network showed in Figure 1 has a frequency reuse factor of 3, and they are $f_{1}, f_{2}$ and $f_{3}$ respectively.

\subsection{Traditional Handover Scenario (without Relay)}

In this scenario, when MS in cell travels along the line in

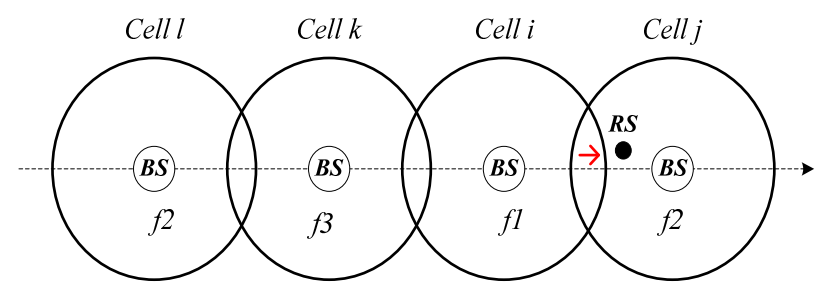

Figure 1. LTE-Advanced network deployment with reuse factor of 3 . 
LTE-Advanced network, it constantly measures the signals from BS in cell (home BS) as well as from BS in cell and cell (neighboring BS). As long as conditions for handover are met, MS handovers are from cell to cell. Without loss of generality, signals transmitted from source to destination can be described as follows [4]:

$$
S_{x}=S r_{x}^{-4} 10^{\left(\xi_{x}+\mu_{x}\right) / 10} \quad x=i, j, k
$$

where $i$ is the ID number of home cell, and $j, k$ are the ID numbers of neighboring cell. $S_{x}$ is the power received from BS in relative cell, $S$ is the transmitted power of the source. $r_{x}$ is the distance between the source and the destination. $\xi_{x}$, which represents the shadowing of signals received from the source, is a Gaussian distributed random variable with a zero mean and a standard deviation $\sigma_{x} \cdot \mu_{x}$ is a constant that represent the Doppler shift effect of signals received from BS or RS in corresponding cells. When MS moves faster, $\mu_{x}$ is getting smaller. $G_{x}$, representing path gain can be defined as follows:

$$
G_{x}=r_{x}^{-4} 10^{\left(\xi_{x}+\mu_{x}\right) / 10} \quad x=i, j, k
$$

The condition for handover in network without relay can be described as follows[5]: when signals received from home BS is at least $\Gamma$ smaller than that received from neighboring $\mathrm{BS}$, then the MS handover to the neighboring BS. The MS handover probability is:

$$
P_{\text {handover }}=\operatorname{Pr}\left[G_{j}-G_{i} \geq \Gamma, G_{j}>G_{k}\right]
$$

Let $\xi=\xi_{0}$, since $\xi$ is a Gaussian distributed random variable with zero mean and a standard deviation $\sigma_{x}$, Equation (3) becomes[6]:

$$
\begin{gathered}
P_{\text {handover }}=\operatorname{Pr}\left[G_{j}-G_{i} \geq \Gamma, G_{j}>G_{k}\right] \\
=\int_{-\infty}^{\infty} \Phi\left(\left[10 \log \left(r_{i} / r_{j}\right)^{4}-\Gamma+\xi_{0}+\left(\mu_{i}-\mu_{j}\right)\right] / \sigma_{i}\right) \\
* \Phi\left(\left[10 \log \left(r_{k} / r_{j}\right)^{4}+\xi_{0}+\left(\mu_{k}-\mu_{j}\right)\right] / \sigma_{k}\right) * \frac{1}{\sqrt{2 \pi} \sigma_{j}} e^{-\frac{\xi_{0}^{2}}{2 \sigma_{j}^{2}}} d \xi_{0}
\end{gathered}
$$

After MS handovers to the target cell, it may be outage because of the co-channel interference and other interference. With the assumption that interfering signals received only from BS in cell $l$ that has the same frequency as cell $j$, the co-channel interference ratio can be represented by:

$$
\frac{C}{I}=\frac{S^{*} r_{j}^{-4} 10^{\left(\xi_{j}+\mu_{j}\right) / 10}}{S * r_{l}^{-4} 10^{\left(\xi_{l}+\mu_{l}\right) / 10}}=\frac{r_{j}^{-4} 10^{\left(\xi_{j}+\mu_{j}\right) / 10}}{r_{l}^{-4} 10^{\left(\xi_{l}+\mu_{l}\right) / 10}}
$$

where $r_{j}, r_{l}$ represent the distance between MS and BS $j$ or BS $l, \xi_{l}$ is a Gaussian distributed variable with zero mean and a standard deviation $\sigma_{l} . \mu_{j}, \mu_{l}$ represent Doppler shift effect of signals received from BS in cell $j$ and in cell $l$, and $\mu_{j}<0, \mu_{l}<0$. The outage probability $P_{\text {target_cell_outage }}$ after handover is given by:

$$
P_{\text {target_cell_outage }}=\operatorname{Pr}\left[\frac{C}{I}<\Delta\right]
$$

where $\Delta$ is a predefined threshold. Let $\xi_{l}=\xi_{0}$, then Equation (6) becomes

$$
\begin{aligned}
& P_{\text {target_cell_outage }} \\
= & \operatorname{Pr}\left[\frac{C}{I}<\Delta\right]=\operatorname{Pr}\left[I^{*} \Delta>C\right] \\
= & \int_{-\infty}^{\infty} F\left(\left[10 \log \left(\frac{\Delta^{\frac{1}{4}} r_{j}}{r_{l}}\right)^{4}+\xi_{0}+\left(\mu_{j}-\mu_{l}\right)\right] / \sigma_{j}\right) \\
& * \frac{1}{\sqrt{2 \pi} \sigma_{l}} * e^{-\frac{\xi_{0}^{2}}{2 \sigma_{l}^{2}}} d \xi_{0}
\end{aligned}
$$

\subsection{LTE-Advanced Handover Scenario (with relay in cells)}

In this scenario, with relay in cells[7], when MS travels along the line, it not only constantly measures signals from home BS and neighboring BS, but also measures signals from the RS that is deployed in cell $j$. In this situation, the handover probability can be divided into two parts: handover to the BS in target cell and handover to the RS in the target cell, which can be given by follows:

$$
\begin{aligned}
& P_{\text {handover_BS }} \\
= & \operatorname{Pr}\left[G_{j}-G_{i} \geq \Gamma, G_{j}>G_{k}, S_{R S}<S_{j}\right] \\
= & \operatorname{Pr}\left[G_{j}-G_{i} \geq \Gamma, G_{j}>G_{k}\right] * \operatorname{Pr}\left[S_{R S}<S_{j}\right] \\
= & P_{\text {handover }} * \operatorname{Pr}\left[a G_{R S}<G_{j}\right] \\
& P_{\text {handover_RS }} \\
= & \operatorname{Pr}\left[S_{R S}-S_{i} \geq S * \Gamma, S_{R S}>S_{k}, S_{R S}>S_{j}\right] \\
= & \operatorname{Pr}\left[S_{R S}-S_{i} \geq S * \Gamma, S_{R S}>S_{k}\right] * \operatorname{Pr}\left[S_{R S}>S_{j}\right] \\
= & \operatorname{Pr}\left[a G_{R S}-G_{i} \geq \Gamma, a G_{R S}>G_{k}\right] * \operatorname{Pr}\left[a G_{R S}>G_{j}\right]
\end{aligned}
$$

Then the total handover probability in the scenario with relay in cells is defined as follows:

$$
P_{\text {handover_sum }}=P_{\text {handover_RS }}+P_{\text {handover_BS }_{-}}
$$

In LTE-Advanced system, frequencies are orthogonal and we assume that the two relay links are distinguished in time division multiplex way. Thus the co-channel interference ratio with BS can be represented as follows:

$$
P_{\text {target_BS_outage }}=\operatorname{Pr}\left[\frac{C}{I}<\Delta\right]=P_{\text {target_cell_outage }}
$$


Considering that the link between BS and RS in LTE-Advanced system is wireless, so the outage probability of the relay access should also involve the BS-RS link, which is given by:

$$
\begin{aligned}
& P_{t a r g e t \_R S_{-} \text {outage }} \\
= & 1-\left(\operatorname{Pr}\left[\frac{C_{R S}}{I_{R S}}>\Delta\right]\right) *\left(\operatorname{Pr}\left[\frac{C_{B S_{-} t o_{-} R S}}{I_{B S_{-} o_{-} R S}}>\Delta_{R S}\right]\right)
\end{aligned}
$$

where $\Delta_{R S}$ is the a predefined threshold for RS, and

$$
\begin{aligned}
\frac{C_{R S}}{I_{R S}} & =\frac{S_{R S} r_{R S}^{-4} 10^{\left(\xi_{R S}+\mu_{R S}\right) / 10}}{S r_{l}^{-4} 10^{\left(\xi_{l}+\mu_{l}\right) / 10}} \\
& =a \frac{r_{R S}^{-4} 10^{\left(\xi_{R S}+\mu_{R S}\right) / 10}}{r_{l}^{-4} 10^{\left(\xi_{l}+\mu_{l}\right) / 10}}
\end{aligned}
$$

The $a$ in Equation (13) represents the transmit power ratio of RS and BS, that is:

$$
\begin{aligned}
& a=\frac{S_{R S}}{S} \\
& \frac{C_{B S_{-} t o_{-} R S}}{I_{B S_{-} t t_{-} R S}}=\frac{r_{B S_{-} t o_{-} R S} S^{-4} 10^{\left(\xi_{j}+\mu_{j}\right) / 10}}{r_{B S_{-}-t o_{-} R S}{ }^{-4} 10^{\left(\xi_{l}+\mu_{l}\right) / 10}}
\end{aligned}
$$

where $r_{\mathrm{BS}_{\perp} \text { to_ } \mathrm{RS}}$ represents the distance between the BS $l$ and the RS and $r_{R S}$, and $r_{l}$ represent the distance between MS and RS or BS $l$.

\section{Numerical Result}

In order to show the impact of RS on handover in highspeed scenarios, we run some simulations using Mathematica and Matlab tools. First we need to take a look at the scenario without RS in the cells.

As can be seen from Figure 2, the handover probability increases as the distance between the BS and MS decreases, but the outage probability after the MS handover to the target BS is opposite, it decreases as the distance between the BS and MS decreases. Seen that handover requests start to increase from about $0.8 \sim 1$ radius from $\mathrm{BS}$, yet the outage probability need to be optimized during this range.

We know that relay technology can improve the system capacity[8] and can also enhance the cell coverage, but whether wireless relay technology in LTE-Advanced system can make contribution to the handover in high speed scenarios is still a question to be studied. In the following, we will make a novel research on this question based on some numerical results that has been deduced in previous analysis.

Compared with the result of Figure 4, there are some differences in Figure 3. In this scenario, RS is deployed in the line that is 0.8 radius away from the BS $j$. When we calculate the outage probability after handover to RS,

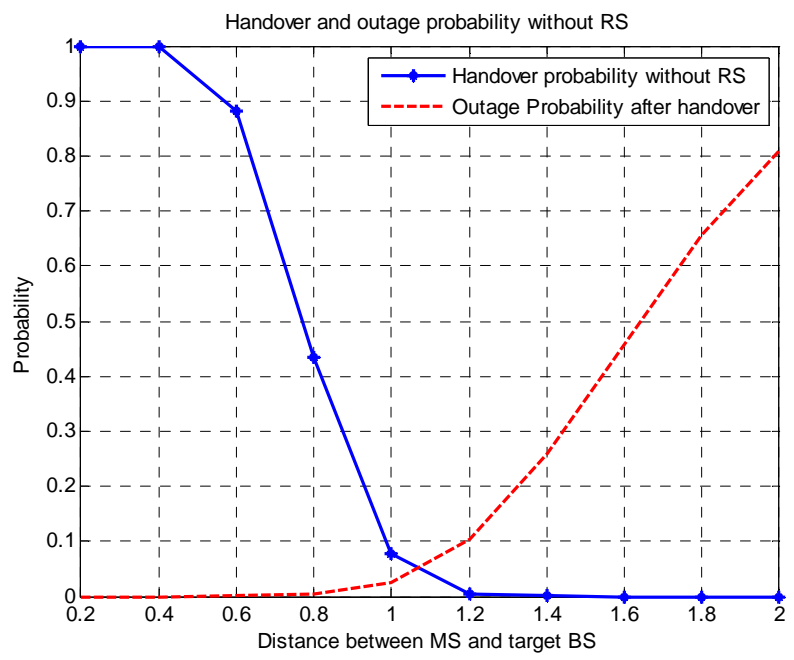

Figure 1. Handover and outage probability without RS.

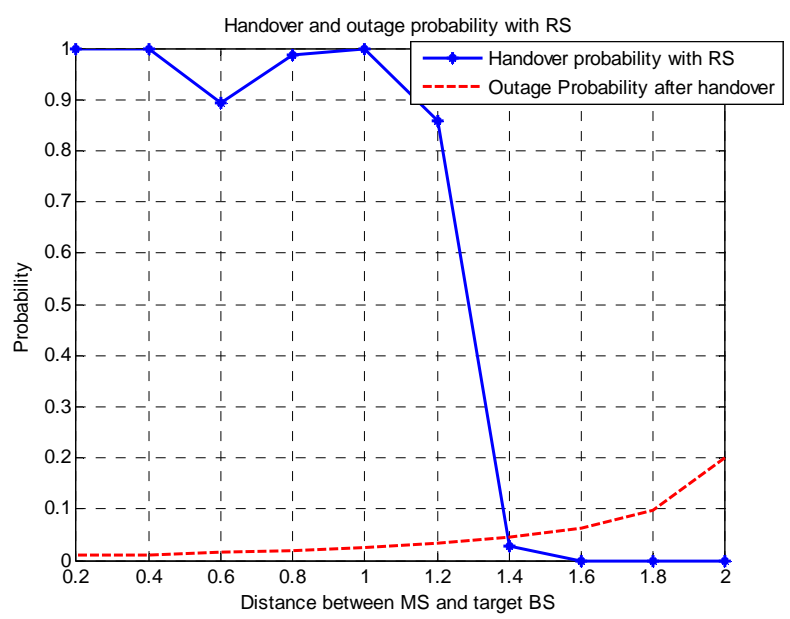

Figure 2. Handover and outage probability with RS.

considering that the BS-RS link is wireless, we calculate both the RS-MS link and the BS-RS link, which is given by Equation (12).

The cells with RS in the boundary do make contributions to the handover outage probability seen from Figure 3, on one hand, it can enlarge the distance that MS is more possible to start a handover, on the other hand, the outage probability is significantly lower and gentler than that without RS.

\section{Conclusions}

In LTE-Advanced systems, coverage in high speed scenario may be a question to be further studied. Based on the previous analysis and some numerical results, we can see that proper relay deployment in the target cell can improve the handover probability distance. For example, in the comparison of Figure 2 and Figure 3, relay deployed in 0.8 radiuses can enlarge about 0.4 radiuses at the same handover probability (0.5). 
At the same time, proper relay deployment can also make the outage probability after handover rise much more slowly as the distances increase, thus it can make the handover more reliable and stable for those in high speed who need wireless communication services.

\section{Acknowledgements}

This paper is supported by Key project (2012ZX03001030-004).

\section{REFERENCES}

[1] R. Pabst, B. Walke, et al., "Relay Based Deployment Concepts for Wireless and Mobile Broadband Radio," IEEE Communications Magazine, pp. 80-89, New York, US, September 2004.

[2] P. Liu, "Solution: How to Overcome the Coverage Issues for WCDMA in High-speed Scenarios,” Huawei Tech- nology, 2006, Vol. 11, pp. 53-55

[3] L. C. Huang and G. Zhu, "Analysis and Optimization of Handover in GSM-R Network,” Mobile Communications, 2007, Vol. 8, pp. 35-38.

[4] A. Molisch, Wireless Communications, Wiley-IEEE Press, 2005.

[5] Z. D. Zhong, X. Li and W. Y. Jiang, "Integrated Digital Mobile Communication System for Railway Transportation (GSM-R) ,” Beijing: China Railway Press, 2003.

[6] D. H. Lee, et al., "Fast Handover Algorithm for IEEE 802.16e Broadband Wireless Access System,” Wireless Pervasive Computing, 2006 1st International Symposium, Jan. 2006.

[7] S. Cho, E. W. Jang and J. M. Cioffi, "Handover in Multihop Cellular Networks,” IEEE Communications Magazine, Vol. 47, 2009, pp. 64-73.

[8] H. Hu, et al., "Range Extension without Capacity Penalty in Cellular Networks with Digital Fixed Relays," Proc. IEEE Globecom ’04, Vol. 5, 2004, pp. 3053-57. 